\title{
Switching protein metalloporphyrin binding specificity by design from iron to
}

\section{fluorogenic zinc}

\author{
B. J. Bowenª, A. R. McGarritya, J-Y. A. Szeto ${ }^{a}$, C. R. Pudney, D. D. Jones ${ }^{a}$
}

a. School of Bioscience, Molecular Biosciences Division, Cardiff University, Cardiff, UK.

b. Department of Biology and Biochemistry, University of Bath, Bath, UK.

\section{Supporting Information}

\section{Supporting Methods.}

\section{In silico design.}

ZincExplorer

In silico protein design was performed by initially using the native wild-type cyt $b_{562}$ sequence with the ZincExplorer web server ${ }^{1}$ (http://protein.cau.edu.cn/ZincExplorer). Successive mutations were assessed by selecting existing residues, replacing them and resubmitting them to the server. The relative scores returned from the server were used to determine if a mutation was likely to improve Zinc protoporphyrin IX (ZnPP) binding (see Figure S1). The first design phase focused on replacing the M7 co-ordinating residue with alternatives to identify a better potential axial ligand residue. The second design round built on the best scoring variant from the first round introduced secondary mutations around residue 7 .

\section{Mutant Modelling}

Using the published crystal structure of wild type cyt $b_{562}\left(\mathrm{PDB}: 256 \mathrm{~B}{ }^{2}\right)$ as a starting point, in silico mutagenesis was performed using the mutagenesis application within MacPyMol ${ }^{3}$ to change a single residue to another. This process was automated using shell scripts to allow for the creation of a library of structure files that replaced residue 1-20 with every other possible residue. Each initial model was used as the starting point and energy minimised to avoid any clashes caused by the mutation introduction. The starting structure was then placed within a triclinic box with dimension of $6.4 \times 6.1 \times 7.8 \mathrm{~nm}$. This was populated using the Simple Point Charge (SPC) water model ${ }^{4}$ to solvate the system to a total number of 16986 solvent molecules. The system was first energy minimised by performing 500 steps of steepest descent method followed by 500 steps of conjugant gradient method. The lowest energy state of the system was used as the starting conformation for the docking simulations.

\section{Ligand Docking with AutoDock}

Structure files for ZnPP were made using Avogadro 5 . Geometry optimization calculations were performed using GAMESS-US ${ }^{6}$ at the HF/6-31G* level in order to be consistent with the AMBER99sb force field ${ }^{7}$. The optimised structure was used as the ligand file during docking simulations. The standard AutoDock ${ }^{8}$ protocol was used as described in the software documentation. The scripts prepare_receptor4, prepare_ligand4, prepare_gpf4, prepare_dpf42 and prepare_flexreceptor4 (distributed with the software) were used to prepare the energy minimised mutated structure files for docking. For each variant 50 models were made and the lowest $5 \%$ 
of the predicted free binding energies were taken and averaged to give an approximate score for each mutation, these were then ranked and the lowest scoring mutant was chosen as the starting point for another round of mutations.

\section{Ligand Docking with Rosetta LigandDock}

The five lowest scoring variants from the AutoDock docking simulations for each round were chosen to be confirmed using the Rosetta LigandDock program ${ }^{9}$. Docking was carried out using the Automatic RosettaLigand Setup script supplied with the program. The predicted binding energies and Rosetta score were compared with the results from the AutoDock docking analysis to inform the choice of which mutation would be used as the starting point of the next round of mutations.

\section{Cyt $b_{562}$ mutagenesis and protein production.}

Construction of cyt $b_{562}{ }^{\mathrm{ZnPP}}$ variants described in this paper was performed using site directed mutagenesis, essentially as outlined previously ${ }^{10}$. Cyt $b_{562}$ and its variants were expressed and purified as outlined previously ${ }^{10}$.

When required, purified cyt $b_{562}$ was converted to apo-form before $\mathrm{ZnPP}$ replacement by using organic solvent extraction approach ${ }^{11}$. The proteins were buffer exchanged into water and the $\mathrm{pH}$ lowered to 1.5 before mixing with ice-cold 2butanone. The mixture was then centrifuged for 2 minutes at 13,000x rpm in a bench top microfuge. The organic phase was removed and the preceding steps were repeated 5 times. The $\mathrm{pH}$ was returned to $\mathrm{pH} 6$ before buffer exchanging back into $10 \mathrm{mM}$ Tris buffer.

ZnPP and hemin was purchased from Sigma Aldrich. The molecules were dissolved in $1 \mathrm{M} \mathrm{NaOH}$ prior to use to a final ZnPP concentration of $1 \mathrm{mM}$. ZnPP was stored in the dark at $4 \mathrm{C}$ and centrifuged prior to use in experiments to prevent molecular aggregation and photodegradation. The ZnPP solution was then added to $10 \mathrm{mM}$ Tris buffer to a final concentration of $200 \mu \mathrm{M}$. This was then added to a solution of $100 \mu \mathrm{M}$ apo- cyt $b_{562}$ in $10 \mathrm{mM}$ Tris buffer and incubated for 12 hours at $25^{\circ} \mathrm{C}$ to form holo cyt $b_{562}$ ZnPP.

\section{Biophysical analysis of cyt $\boldsymbol{b}_{562}$ variants}

UV-Vis Spectrophotometry and porphyrin titration

The absorbance spectra were monitored using Cary UV-Vis Spectrophotometer. The titration experiments were performed using $20 \mu \mathrm{M}$ apo-cyt $b_{562}$ in $1 \mathrm{ml}$ of $10 \mathrm{mM}$ phosphate buffer $\mathrm{pH}$ 6.2. Either haemin $(20 \mu \mathrm{M}$ stock) or $\mathrm{ZnPP}(20 \mu \mathrm{M}$ stock) in the were titrated against the protein solution. Apo-cyt $b_{562}$ concentration was determined by measuring the absorbance at $280 \mathrm{~nm}$ (Molar absorbance coefficient $2.98 \mathrm{mM}^{-1}$ $\mathrm{cm}^{-1}$ ). Binding of metalloporphyrin to apo-protein was measured by monitoring the increase in absorbance at either $430 \mathrm{~nm}$ for ZnPP titration or $418 \mathrm{~nm}$ for hemin. The resultant titration curves were then fitted to $\mathrm{Eq} 1$ below to determine dissociation constant, as described previously 1012 .

$$
\begin{aligned}
& \text { Eq } 1 \\
& {[P]_{t}=\frac{[P]_{0} V_{0}}{V_{0}+V_{L}} \quad[L]_{t}=\frac{[L]_{0} V_{L}}{V_{0}+V_{L}}}
\end{aligned}
$$


$\Delta \mathrm{A}=\frac{C}{2}\left[\left([P]_{t}+[L]_{t}+K_{d}\right)-\sqrt{\left([P]_{t}+[L]_{t}+K_{d}\right)^{2}-4[P]_{t}[L]_{t}}\right]$

where $\Delta \mathrm{A}$ is change in absorbance, $C$ is the molar absorbance coefficient, $[\mathrm{P}]_{t}(\mu \mathrm{M})$ and $[\mathrm{L}] \mathrm{t}(\mu \mathrm{M})$ are the protein and protoporphyrin total concentrations and $K_{d}$ is the dissociation constant. The total concentration of protein and its ligand can be obtained considering the initial volume $\left(\mathrm{V}_{0}, \mu \mathrm{l}\right)$ and concentration $\left([\mathrm{P}]_{0}, \mu \mathrm{M}\right)$ of the protein solution, the concentration of the protoporphyrin solution $\left([\mathrm{L}]_{0}, \mu \mathrm{M}\right)$ and the total volume of the ligand $(\mathrm{V} L, \mu \mathrm{l})$ added during the experiment:

\section{Fluorescence Spectroscopy}

Excitation and emission spectra were recorded using a Varian Cary Eclipse spectrophotometer and the corresponding Cary Eclipse software. Samples of $750 \mu \mathrm{L}$ comprising of 2.5-10 $\mu \mathrm{M}$ protein/porphyrin were analysed in a $5 \times 5 \mathrm{~mm}$ QS quartz cuvette (Hellma, Müllheim, Germany). Spectra were recorded at a scan rate of either 120 or $600 \mathrm{~nm} / \mathrm{min}$ with a slit width of $5 \mathrm{~nm}$. Emission spectra were recorded up to $700 \mathrm{~nm}$ from a fixed excitation wavelength (corresponding to $\lambda_{\mathrm{MAX}}$ from the absorbance spectrum [ 430 $\mathrm{nm}$ and $\sim 590 \mathrm{~nm}$ ] with an associated emission peak) for each variant. Quantum Yield studies were carried out using Alexa Fluor 431, essentially as described previously ${ }^{13-14}$. For the in vivo analysis, liquid cell cultures were grown at $37^{\circ} \mathrm{C}$ to an $A_{600}$ of 0.6 . The cells were pelleted by centrifugation in a microfuge for $20 \mathrm{~min}$ at $4^{\circ} \mathrm{C}$. $\mathrm{ZnPP}(20 \mu \mathrm{M})$ in $10 \mathrm{mM}$ Tris- $\mathrm{HCl}(\mathrm{pH} 8.0)$ was used to resuspended the cell pellet. A cell culture without the plasmid encoding the cyt $b_{562}$ variants was used as a control. Fluorescence readings were taken periodically to determine rate of ZnPP uptake and incorporation. Protein affinity for ZnPP was also performed by fluorescence. ZnPP was titrated into $5 \mu \mathrm{M}$ of protein and fluorescence measured as above. Each titration was performed in triplicate. The fluorescence increase at $597 \mathrm{~nm}$ was extracted and fitted to an equation for fluorescent ligands binding to protein, as described by Motulsky and Neubig ${ }^{15}$. Data was fitted using Graphpad Prism.

\section{Red edge excitation spectra (REES) analysis}

All fluorescence measurements were performed using a Varian Cary Eclipse fluorescence spectrophotometer (Agilent Technologies) with a $5 \times 5 \mathrm{~mm}$ QS quartz cuvette (Hellma UK). The fluorimeter was baselined with $10 \mathrm{mM}$ sodium phosphate buffer ( $\mathrm{pH} 6.2)$ and all measurements were taken at $20^{\circ} \mathrm{C}$. Excitation and emission slit widths were $5 \mathrm{~nm}$ and a scan rate of 600 or $120 \mathrm{~nm} / \mathrm{min}$ was used. Samples were incubated for 30 minutes at the given conditions prior to recording measurements. The red edge excitation scans were monitored from 413 to $437 \mathrm{~nm}$ with increments of $2 \mathrm{~nm}$ in the excitation wavelength. The wavelength of maximum emission, was extracted from the emission spectra by fitting to a sum (two) of skewed Gaussians of the form:

$$
F I=F I_{0}+A e^{-e^{-\left(\frac{\lambda_{E m}-\lambda_{E m}^{\max }}{w}\right)}-\frac{\left(\lambda_{E m}-\lambda_{E m}^{\max }\right)}{w}+1} \quad \text { Eq } 2
$$

where $\mathrm{Fl}$ is the measured fluorescence intensity at an emission wavelength, $\lambda_{\mathrm{EM}}$. The spectra are then characterised by the amplitude, $\mathrm{A}$ of the spectrum at the maximum of fluorescence intensity, $\lambda_{\mathrm{EM}}{ }^{\max }$, spectral width at half 
maximal, $\mathrm{w}$ and the minimum of the fluorescence emission, $\mathrm{Fl}_{0}$, which was $\sim$ zero for the buffer subtracted spectra.

CD spectroscopy

Circular dichroism spectroscopy was performed on a Jasco J-710 as described previously ${ }^{12}$. 
Table S1. Metalloporphyrin affinity of cyt $b_{562}$ variants

\begin{tabular}{|l|c|c|c|c|}
\hline & \multicolumn{2}{|c|}{$\begin{array}{c}\text { Predicted } \\
\text { Affinity (kcal/mol) }\end{array}$} & \multicolumn{2}{c|}{ Measured Affinity (nM) } \\
\hline Variant & ZnPP & Haem & ZnPP & Haem \\
\hline Wild Type & -8.7 & -12.3 & $\begin{array}{l}404^{\mathrm{b}} \\
626^{\mathrm{c}}\end{array}$ & $10^{\mathrm{e}}$ \\
\hline cyt $b_{562}$ M7C & -8.9 & -8.9 & $181^{\mathrm{b}}$ & $206^{\mathrm{b}}$ \\
\hline cyt $b_{562^{2 n P P}}$ & -9.5 & -9 & $32^{\mathrm{c}, \mathrm{d}}$ & $\mathrm{ND}^{\mathrm{f}}$ \\
\hline
\end{tabular}

${ }^{\mathrm{a}}$ calculated using Autodock. ${ }^{8}$; ${ }^{\mathrm{b}}$ calculated using absorbance method (see Figure S3b-c); ${ }^{c}$ calculated using fluorescence method (see Figure $2 \mathrm{a}$ in main paper); ${ }^{\mathrm{d}}$ affinity too tight to measure accurately by current approaches so an upper estimate is given (see Figure S3c). The fluorescence binding curve shown in Figure 2 in the main text clearly shows maximal binding is reached at an equimolar concentration of protein and ligand. Curve fitting generates a $K_{D}$ of $34 \mathrm{nM} \pm 10$. A similar trend is seen with the absorbance approach (Figure S3c) with an estimated $K_{\mathrm{D}}$ of $1 \mathrm{nM}$. e determined previously ${ }^{12}$ and independently verified here as $16 \mathrm{nM} \pm 1 ;{ }^{f}$ affinity too weak to measure.

Table S2. Binding affinities for additional cyt $b_{562}$ variants for ZnPP and haem.

\begin{tabular}{|l|c|c|}
\hline \multicolumn{1}{|c|}{ Variant } & \multicolumn{2}{c|}{$\begin{array}{c}\text { Measured binding affinity } \\
\text { (nM) }\end{array}$} \\
\cline { 2 - 3 } & ZnPP & Haem \\
\hline cyt $b_{562} \mathrm{M} 7 \mathrm{H}$ & 458 & 100 \\
\hline cyt $b_{562} \mathrm{M} 7 \mathrm{G}$ & 409 & 472 \\
\hline cyt $_{5662}{ }^{\mathrm{nPP}} \mathrm{L}(\mathrm{R}) 10 \mathrm{~A}$ & 152 & 200 \\
\hline cyt $b_{562}{ }^{\mathrm{nPP}} \mathrm{L}(\mathrm{R}) 10 \mathrm{~S}$ & 108 & 248 \\
\hline
\end{tabular}




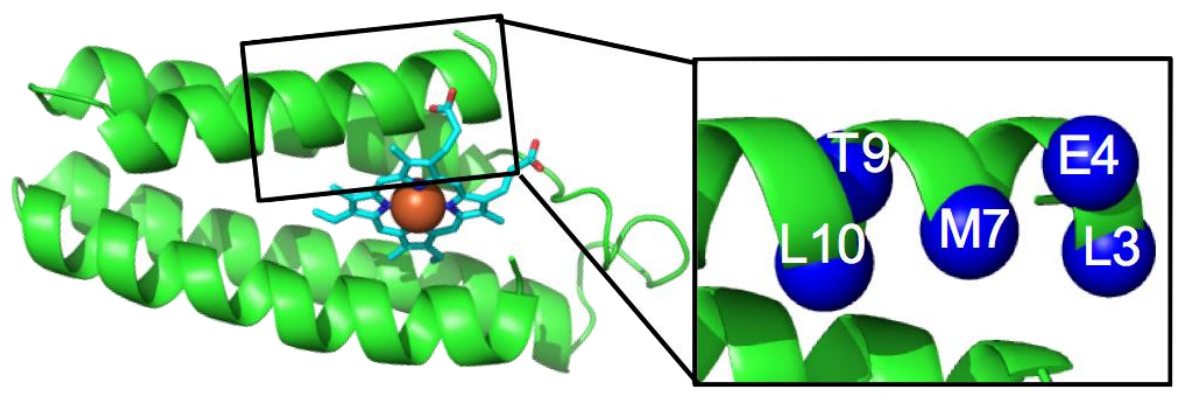

Figure S1. Cytochrome $b_{562}$ structure. The protein is shown as green cartoon with haem shown in the stick representation and coloured cyan; the Fe centre is coloured brown. On the right hand side, the residues targeted for mutagenesis in helix $\mathrm{H} 1$ are shown.

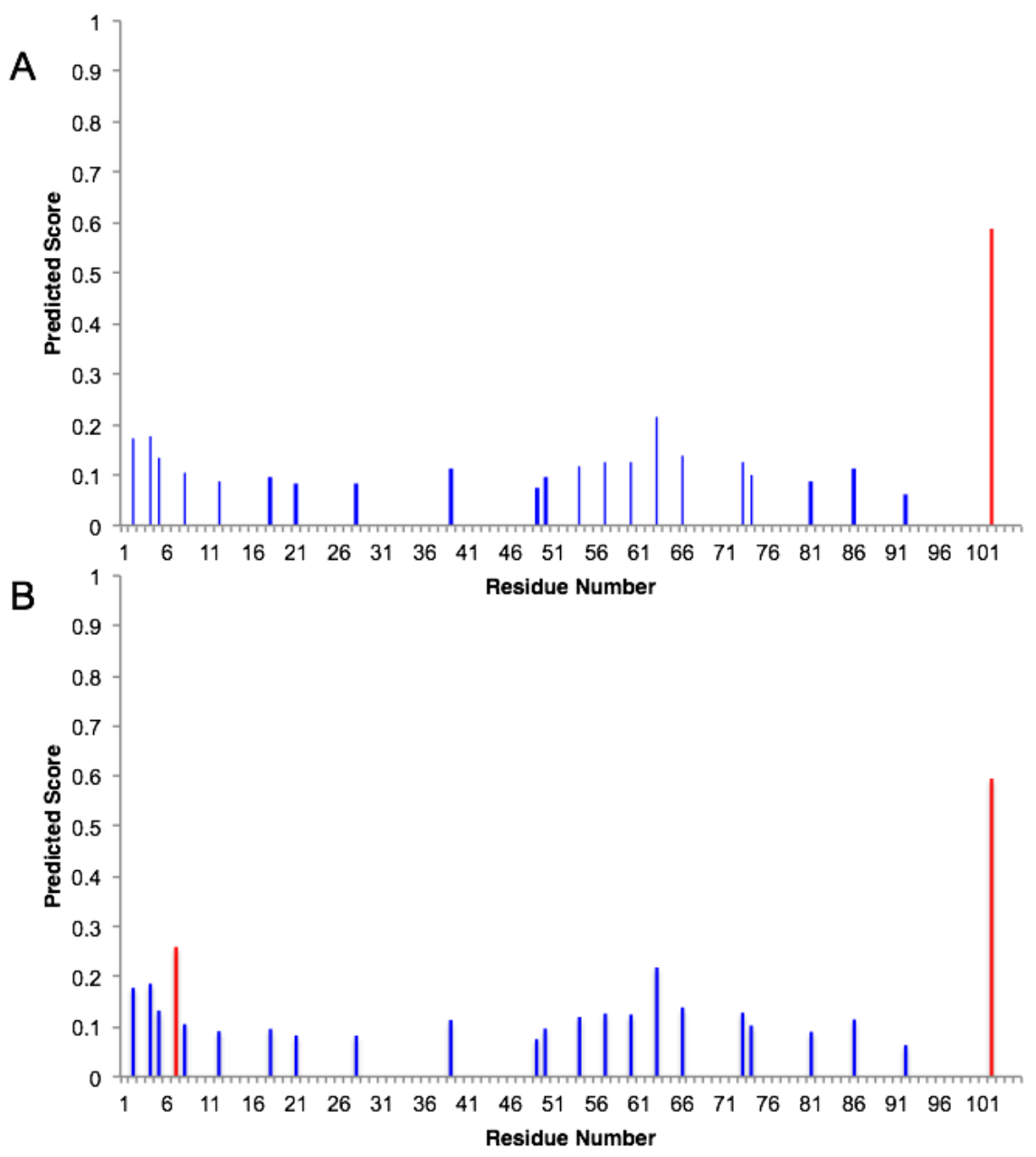

Figure S2. Prediction of $\mathrm{Zn}^{2+}$ binding sites for (A) wild-type cyt $b_{562}$ and (B) cyt $b_{562}{ }^{\mathrm{M} 7 \mathrm{C}}$. Lines highlighted in red show the greatest propensity to bind $\mathrm{Zn}^{2+}$. 


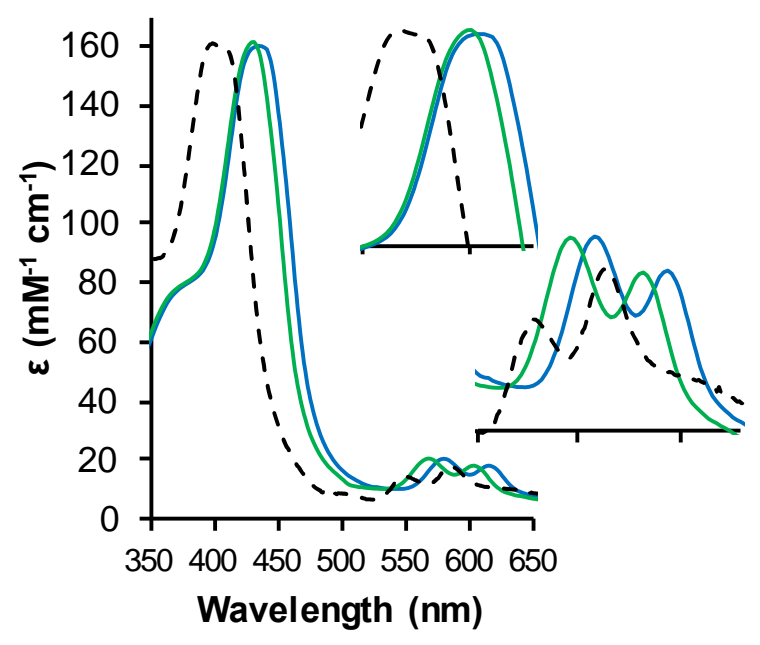

Figure S3. Absorbance spectra of free ZnPP (black dashed line) and bound to wt cyt $b_{562}$ (blue line) and cyt $b_{562}{ }^{\mathrm{M} 7 \mathrm{C}}$. Inset are enlarged images of the Soret peak (top) and $\alpha / \beta$ band (bottom). 

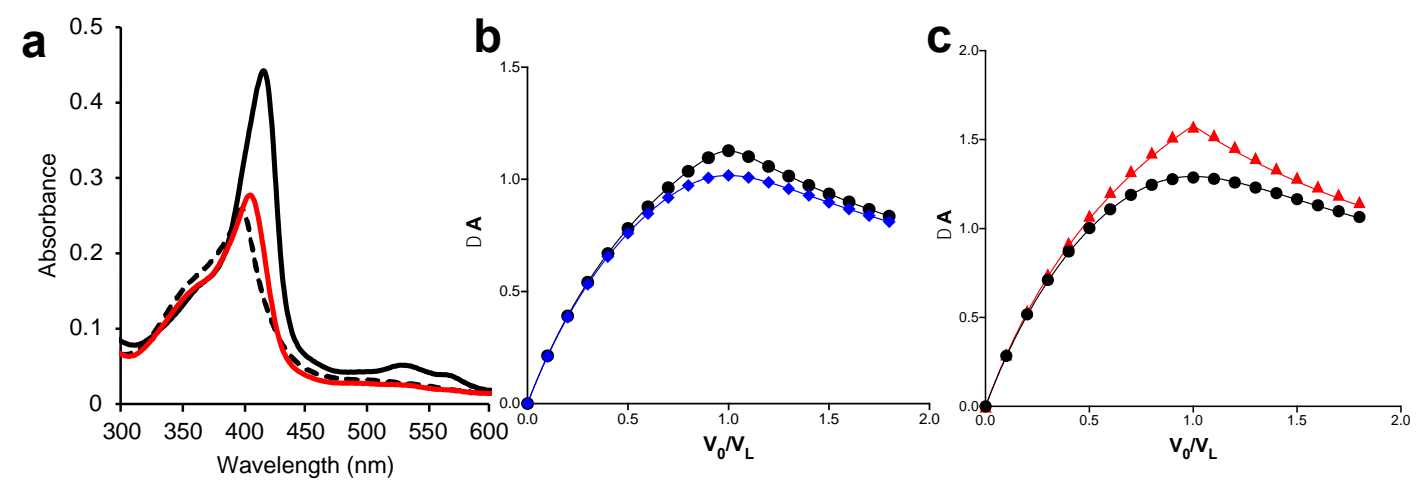

Figure S4. Binding affinity for haem and ZnPP. (a) cyt $b_{562}{ }^{\mathrm{ZnPP}}$ (red) and wt cyt $b_{562}$ (black) haem binding capacity. Protein $(5 \mu \mathrm{M})$ was incubated with $6 \mu \mathrm{M}$ haem and binding monitored. The free haem spectrum (dashed black lines) is shown for reference. Less than $10 \%$ haem binding to cyt $b_{562} \mathrm{ZnPP}$ was observed. (b) Haem and (c) ZnPP titration curves. The wild-type cyt $b_{562}$ (black line, circles), cyt $b_{562}{ }^{\mathrm{M} 7 \mathrm{C}}$ (blue line, diamonds) cyt $b_{562}{ }^{\mathrm{nPP}}$ (red) are shown. The data is based on triplicate reading (errors bars are removed for clarity). The data was fit and the $K_{\mathrm{D}}$ was calculated using a previously described approach ${ }^{10,12}$.

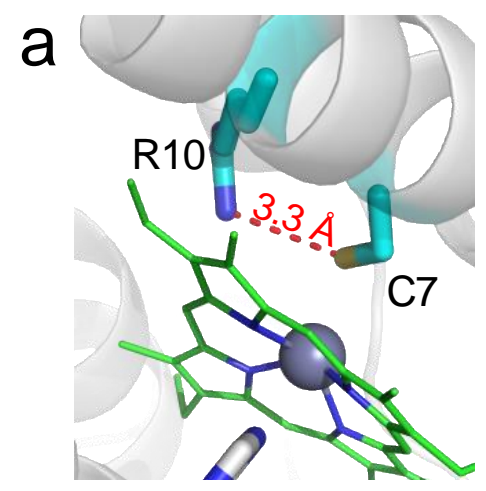

b

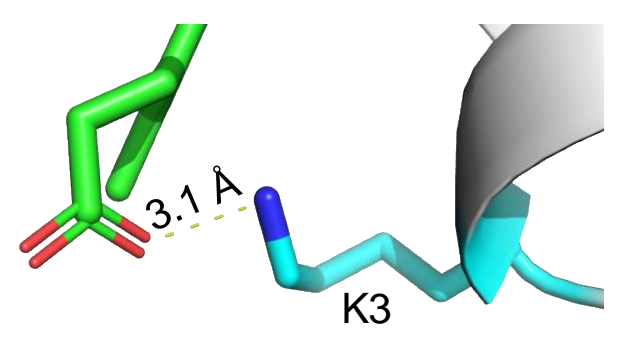

Figure S5. Model of the cyt $b_{562} \mathrm{ZnPP}$ binding pocket. (a) The distance and relative position of the introduced guanidinium group of R10 and the new thiol group that can coordinate $\mathrm{Zn}^{2+}$ is shown as a red dashed line. (b) Putative salt-bridge bond between K3 and carboxyl group of ZnPP. 


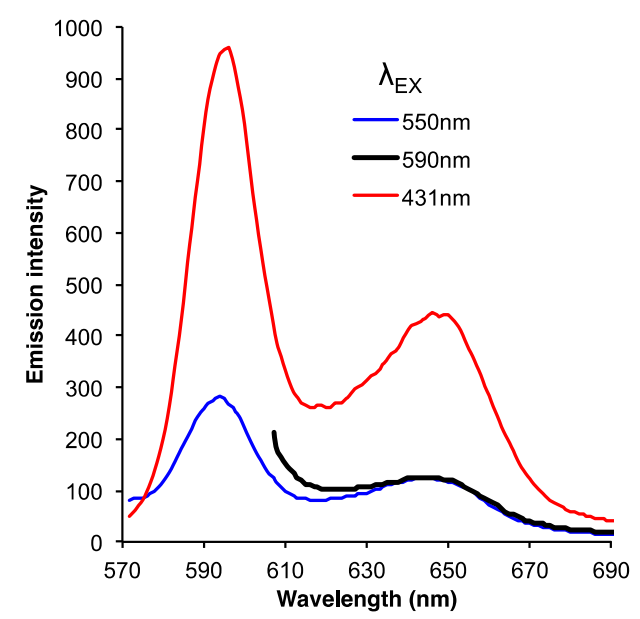

Figure S6. Relative fluorescence emission of profiles of ZnPP bound to cyt $b_{562^{\mathrm{ZPP}}}$ on excitation at the wavelengths stated in the figure.

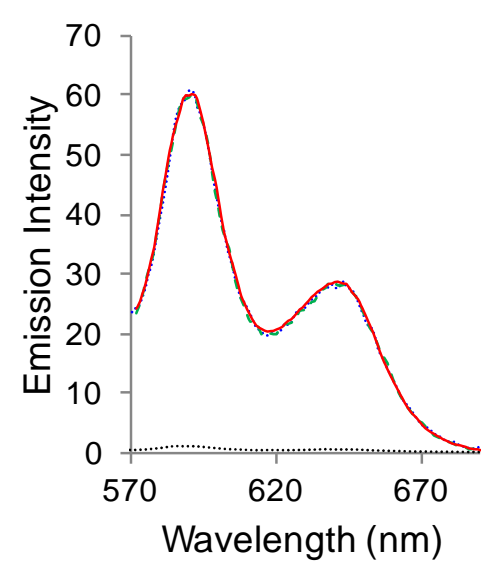

Figure S7. Fluorescence emission profile for cyt $b_{562}$ variants bound to ZnPP. Red line, cyt $b_{562}{ }^{\mathrm{nPP}}$; dashed green line, cyt $b_{562}{ }^{\mathrm{M} 7 \mathrm{C}}$; blue dotted line, wt cyt $b_{562}$; dotted black line free ZnPP.

\section{Supporting References.}

1. Chen, Z.; Wang, Y.; Zhai, Y. F.; Song, J.; Zhang, Z., ZincExplorer: an accurate hybrid method to improve the prediction of zinc-binding sites from protein sequences. Mol Biosyst 2013, 9 (9), 2213-22.

2. Lederer, F.; Glatigny, A.; Bethge, P. H.; Bellamy, H. D.; Matthew, F. S., Improvement of the $2.5 \mathrm{~A}$ resolution model of cytochrome b562 by redetermining the primary structure and using molecular graphics. Journal of molecular biology 1981, 148 (4), 427-48.

3. Schrodinger, LLC, The PyMOL Molecular Graphics System, Version 1.3r1. 2010.

4. Berweger, C. D.; van Gunsteren, W. F.; Müller-Plathe, F., Force field parametrization by weak coupling. Re-engineering SPC water. Chemical Physics Letters 1995, 232 (5), 429-436.

5. Hanwell, M. D.; Curtis, D. E.; Lonie, D. C.; Vandermeersch, T.; Zurek, E.; Hutchison, G. R., Avogadro: an advanced semantic chemical editor, 
visualization, and analysis platform. Journal of cheminformatics 2012, 4 (1), 17.

6. Schmidt, M. W.; Baldridge, K. K.; Boatz, J. A.; Elbert, S. T.; Gordon, M. S.; Jensen, J. H.; Koseki, S.; Matsunaga, N.; Nguyen, K. A.; Su, S.; Windus, T. L.; Dupuis, M.; Montgomery, J. A., General atomic and molecular electronic structure system. Journal of Computational Chemistry 1993, 14 (11), 13471363.

7. Hornak, V.; Abel, R.; Okur, A.; Strockbine, B.; Roitberg, A.; Simmerling, C., Comparison of multiple Amber force fields and development of improved protein backbone parameters. Proteins 2006, 65 (3), 712-25.

8. Morris, G. M.; Huey, R.; Lindstrom, W.; Sanner, M. F.; Belew, R. K.; Goodsell, D. S.; Olson, A. J., AutoDock4 and AutoDockTools4: Automated docking with selective receptor flexibility. J Comput Chem 2009, 30 (16), 2785-91.

9. Meiler, J.; Baker, D., ROSETTALIGAND: protein-small molecule docking with full side-chain flexibility. Proteins 2006, 65 (3), 538-48.

10. Della Pia, E. A.; Macdonald, J. E.; Elliott, M.; Jones, D. D., Direct binding of a redox protein for single-molecule electron transfer measurements. Small 2012, 8 (15), 2341-4.

11. Jones, D. D.; Barker, P. D., Design and characterisation of an artificial DNAbinding cytochrome. Chembiochem 2004, 5 (7), 964-71.

12. Della Pia, E. A.; Chi, Q.; Elliott, M.; Macdonald, J. E.; Ulstrup, J.; Jones, D. D., Redox tuning of cytochrome b562 through facile metal porphyrin substitution. Chemical communications 2012, 48 (86), 10624-6.

13. Arpino, J. A.; Rizkallah, P. J.; Jones, D. D., Crystal structure of enhanced green fluorescent protein to 1.35 a resolution reveals alternative conformations for glu222. PloS one 2012, 7 (10), e47132.

14. Reddington, S. C.; Driezis, S.; Hartley, A. M.; Watson, P. D.; Rizkallah, P. J.; Jones, D. D., Genetically encoded phenyl azide photochemistry drives positive and negative functional modulation of a red fluorescent protein. RSC Advances 2015, 5, 77734-77738.

15. Motulsky, H. J.; Neubig, R. R., Analyzing binding data. Curr Protoc Neurosci 2010, Chapter 7, Unit 75. 